\title{
CORRELAÇÃO DO TÔNUS MUSCULAR E DA AMPLITUDE DE MOVIMENTO EM CADEIA CINÉTICA FECHADA DE TORNOZELO NO EQUILÍBRIO DE INDIVÍDUOS PÓS-ACIDENTE VASCULAR ENCEFÁLICO
}

Correlation of muscle tone and movement amplitude in ankle closed kinetic

chain in post-vascular encephalic individuals balance

\author{
Bruna Alegre Viana ${ }^{1}$; ReniVolmir Dos Santos ${ }^{2}$
}

${ }^{1}$ Fisioterapeuta - Universidade Luterana do Brasil - ULBRA - Canoas/RS. E-mail: brualegreviana@, gmail.com

${ }^{2}$ Docente do Curso de Fisioterapia da Universidade Luterana do Brasil - ULBRA - Canoas/RS. E-mail: revols@uol.com.br

Data do recebimento: 15/10/2020 - Data do aceite: 11/01/2021

RESUMO: O Acidente Vascular Encefálico (AVE) é um déficit neurológico focal devido a infarto ou hemorragia de início rápido e duração de mais de 24 horas, sendo um dos principais problemas de saúde pública mundial. Déficits nas funções dos membros inferiores frequentemente persistem na grande maioria dos casos, afetando a marcha, o equilíbrio e a mobilidade funcional. O objetivo do estudo foi de verificar a correlação do tônus muscular e da amplitude de movimento em cadeia cinética fechada de tornozelo no equilíbrio de indivíduos pós-AVE. A amostra de 14 indivíduos hemiparéticos, com diagnóstico de AVE, foram avaliados pela escala de Ashworth modificada, escala de Equilíbrio de Berg, DorsiflexionLunge Test (DLT) e a Goniometria, num estudo transversal. A média de idade foi de 59,07( $\pm 14,93)$ anos e com tempo de diagnóstico de 86,57( $\pm 60,67)$ meses, sendo 9 homens e 5 mulheres. Na correlação da escala de equilíbrio de Berg com o tônus muscular não houve correlação significativa, porém com a amplitude de movimento do tornozelo esta foi estatisticamente significativa, sendo DLT em centímetros $\mathrm{r}=0,502$ e $p=0,046$; DLT graus $r=0,488$ e $p=0,046$; Goniometria $r=0,472$ e $p=0,049$; 
e flexometria $\mathrm{r}=0,490$ e $\mathrm{p}=0,048$. Conclui-se que há correlação positiva da amplitude de tornozelo com o equilíbrio, na amostra estudada.

Palavras-chave: Acidente vascular encefálico. Equilíbrio. Tornozelo. Amplitude articular. Tônus muscular.

ABSTRACT: Stroke is a focal neurological deficit due to a fast onset infarction or hemorrhage lasting more than 24 hours, it is one of the main worldwide public health problems. Deficits in lower limb functions often persist in most cases, affecting gait, balance and functional mobility. The objective of this study was to verify the correlation of muscle tone and range of motion in ankle closed kinetic chain in the balance of post-stroke individuals. The sample consisted of 14 hemiparetic individuals, diagnosed with stroke, they were assessed using the modified Ashworth scale, Berg Balance Scale, Dorsiflexion Lunge Test (DLT) and Goniometry, in a cross-sectional study. The mean age was $59.07( \pm 14.93)$ years old and with a diagnosis time of $86.57( \pm 60.67)$ months, 9 men and 5 women. The correlation of the Berg balance scale with muscle tone was not significant, however with the range of motion of the ankle was statistically significant, with DLT in centimeters $r=0,502$ and $p=0,046$; DLT degrees $r=0,488$ and $p=0,046$; Goniometry $r=0,472$ and $p=0,049$; and Flexometry $r=0,490$ and $p=0,048$. It was concluded that there is a positive correlation between ankle amplitude chain and balance, in the studied sample.

Keywords: Stroke. Balance. Ankle. Joint amplitude. Muscle tone.

\section{Introdução}

Segundo a Organização Mundial de Saúde (OMS), o Acidente Vascular Encefálico (AVE) é uma alteração neurológica de rápido desenvolvimento, consequente de uma interrupção do fluxo sanguíneo em alguma determinada área encefálica, a interrupção pode ocorrer por hemorragia ou isquemia (BRASIL, 2016).

As sequelas neurológicas deixadas pelo AVE no indivíduo afetam diferentes áreas e funções de seu corpo, como a função motora, que é a capacidade do corpo produzir um movimento voluntário ou automático (BROUSSY et al., 2019). A marcha hemiplégica ou hemiparética surge com a perda ou comprometimento unilateral das funções sensoriais e motoras reduzindo a capacidade do corpo em transferir seu peso, e também da capacidade de suporte do membro inferior do lado acometido, o que resulta numa marcha assimétrica, pois os indivíduos diminuem o tempo da fase de apoio do membro afetado, e descarregam a maior parte de seu peso corporal para o membro não afetado (SZOPA et al., 2017; MATSUDA et al., 2017; HOLT et al., 2019).

Após o AVE, a deficiência neurológica depende da dimensão e localização da lesão. As alterações de equilíbrio, motoras, sensitivas, assim como déficit cognitivo e da linguagem, são as principais ocorrências que acometem o indivíduo. Dentre as apresentações, a hemiparesia é a mais comum, ocasionando déficits motores no hemicorpo acometido, podendo exibir, alteração do tônus, falta de estabilida- 
de e coordenação, diminuição da amplitude de movimento, entre outras (DORNELLES; OLIVIERA, 2019; SAVASSINI et al., 2019). Alterações no tônus (espasticidade) e na força muscular, podem acometer os flexores plantares e dorsais do tornozelo, que desempenham papel fundamental no ortostatismo, nas fases e no padrão da marcha, bem como nas estratégias de equilíbrio estático e dinâmico (MEULEN, 2016) e que pode levar a quedas (MEDEIROS et al., 2019).

A diminuição da simetria e a dificuldade nas transferências de peso na postura em pé também podem alterar o equilíbrio, a marcha e a função motora em geral (NEVES et al., 2011; LENDRAITIENE et al., 2017). Assim, a identificação dos déficits proporciona um tratamento apropriado, sendo o ponto mais difícil no processo de planejamento do trabalho, necessitando da capacidade profissional do fisioterapeuta em avaliar os dados apresentados (MOLITERNO et al., 2019; CACHO et al., 2017).

A Escala de Equilíbrio de Berg (EEB) apresentou uma boa sensibilidade para identificar o risco de queda, assim como o equilíbrio (SONG; LEE; SHIN, 2018) em indivíduos pós-AVE (ALVES et al., 2017), enquanto a avaliação do tônus ocorre por escalas ordenadas como a de Ashworth modificada, considerada padrão ouro para mensuração clínica do tônus muscular em indivíduos com AVE (CHEN et al., 2019). Em Cadeia Cinética Fechada (CCF) observam-se movimentos multiarticulares executados com a extremidade distal fixa, frequentemente com descarga de peso associada. Uma das avaliações utilizadas em CCF é o Lunge Test que quantifica a dorsiflexão de tornozelo (HALL; DOUCHERTY, 2017).

As avaliações que quantificam essas alterações são de grande importância para elaboração de tratamentos e para constatar a progressão dos pacientes. Portanto, a veri- ficação da amplitude de movimento (ADM) da articulação de tornozelo tem extrema importância, pois em $70 \%$ dos hemiparéticos se observa alteração nesta articulação (KIM; LEE, 2018). Também devido a espasticidade dos plantiflexores de tornozelo, há uma diminuição da ADM para o movimento de dorsiflexão gerando uma possível alteração no equilíbrio, pois a perda de amplitude dessa articulação dificulta as reações de endireitamento e equilíbrio para a manutenção da postura. Diante do exposto, o presente estudo objetivou a associação do tônus muscular e da amplitude de movimento em CCF da articulação de tornozelo no equilíbrio de indivíduos pós-AVE.

\section{Materiais e métodos}

Estudo do tipo transversal, realizado no período de agosto a setembro de 2019, em uma Clínica-Escola da região metropolitana de Porto Alegre/RS. Esta pesquisa foi realizada de acordo com as diretrizes da Resolução 466/12 do Conselho Nacional de Saúde do Ministério da Saúde e foi aprovada pelo Comitê de Ética em Pesquisa da Universidade Luterana do Brasil - Campus de Canoas/ RS sob o parecer o $\mathrm{n}^{\circ} 3.423 .267$, CAAE 14236919.6.0000.5349.

Foram incluídos no estudo indivíduos com diagnóstico clínico de AVE, com idade superior a 18 anos e em fase crônica (maior que 6 meses pós-ictus), com diagnóstico de AVE isquêmico ou hemorrágico e quadro de hemiparesia, totalizando 14 participantes, que assinaram o Termo de Consentimento Livre e Esclarecido (TCLE).

Inicialmente, os participantes da pesquisa responderam uma ficha de identificação, contendo nome, idade, tempo de diagnóstico, hemicorpo acometido e o tipo de AVE.

A avaliação do tônus muscular foi através da escala de Ashworth modificada, que é a 
mais citada na literatura para avaliação do tônus muscular em pacientes que apresentam disfunção do sistema nervoso central. É uma escala para avaliação do grau de espasticidade, que é medida de acordo com a resistência oferecida ao movimento angular de um segmento movido de forma rápida e passiva por um examinador. Essa escala avalia o tônus em graus de 0-4. E classifica o tônus conforme a restrição à movimentação passiva dos grupos musculares de membros superiores e inferiores, graduando conforme a escala, maior o grau, maior o nível da hipertonia (espasticidade) foi testada por meio do alongamento passivo da musculatura. Os músculos avaliados foram o gastrocnêmio e o sóleo. O plantiflexorsóleo foi avaliado, com o indivíduo em decúbito lateral com flexão de quadril a $45^{\circ}$ e flexão de joelho em $45^{\circ}$ o terapeuta com uma das mãos estabiliza a articulação do tornozelo e com a outra realizou uma dorsiflexão passiva partindo de uma plantiflexão. Para avaliar o gastrocnêmio, o posicionamento foi em decúbito lateral com flexão de quadril a $45^{\circ}$ e extensão completa de joelho o terapeuta realizou uma dorsiflexão passiva e com a outra mão estabilizou a perna do paciente (BLACKBURN; VAN VLIET; MOCKETT, 2002).

Para a avaliação da ADM do tornozelo em CCF foi usado o teste Lunge Test (LT), também conhecido como Weight-BearingLunge Test (WBLT). Este teste é feito contra uma parede e aplicado bilateralmente. Uma fita métrica padrão $(\mathrm{cm})$ é necessária e o flexímetro. O teste foi iniciado numa posição standard de $10 \mathrm{~cm}$ de distância da parede. A distância foi aumentada ou diminuída $1 \mathrm{~cm}$ de cada vez até se encontrar a distância máxima em que o participante conseguiu tocar com o joelho na parede mantendo o calcâneo a tocar no chão. Foi dada a indicação aos participantes que podiam apoiar-se na parede com os membros superiores e ter o membro inferior contralateral numa posição confortável. Isso coloca a articulação do tornozelo em dorsiflexão máxima. A distância máxima da parede até a ponta do hálux do pé foi registrada. $\mathrm{O}$ flexímetro foi posicionado na tuberosidade da tíbia para medir a ADM. A distância é medida em centímetros $(\mathrm{cm})$ com cada centímetro correspondente a aproximadamente $3,6^{\circ}$ de dorsiflexão do tornozelo. Sendo uma medida válida e confiável da ADM de dorsiflexão de tornozelo (HALL; DOUCHERTY, 2017).

Neste momento foi realizada a avaliação do dorsiflexão do tornozelo através da goniometria. Para a análise da ADM foi utilizado um goniômetro universal. Os pontos de referência foram marcados, a linha mediana do braço fixo do goniômetro ficou posicionada sobre a fíbula e a linha externa do braço móvel posicionada sobre a cabeça do quinto metatarso. O fulcro do goniômetro foi secundariamente posicionado abaixo do maléolo lateral (eixo da articulação). O tornozelo foi colocado em posição neutra e o voluntário foi instruído a fazer o movimento de dorsiflexão ativa do tornozelo, enquanto trazia a tíbia a frente (GOUVEIA et al., 2014).

Finalizando, os participantes da pesquisa foram avaliados quanto ao equilíbrio através da Escala de Equilíbrio de Berg (Berg Balance Scale). Essa escala foi criada em 1992 por Katherine Berg, amplamente utilizada para avaliar o equilíbrio nos indivíduos em 14 itens comuns à vida diária. Cada item possui uma escala ordinal de cinco alternativas que variam de 0 a 4 pontos. Portanto, a pontuação máxima pode chegar a 56. Os pontos são baseados no tempo em que uma posição pode ser mantida, na distância em que o membro superior é capaz de alcançar à frente do corpo e no tempo para completar a tarefa. Para a realização são necessários um relógio, uma régua, um banquinho e duas cadeiras, e o tempo de execução é de aproximadamente 15 minutos. Assim, cada ponto a menos na escala corresponde a um aumento do risco de quedas: entre os escores 56 a 54, cada 
ponto a menos é associado a um aumento de 3 a $4 \%$ no risco de quedas; entre 54 e 46 , a um aumento de 6 a $8 \%$ de chances, sendo que abaixo de 36 pontos o risco de quedas é de quase $100 \%$ (MIYAMOTO et al., 2004).

O tamanho da amostra foi calculado usando o software G*Power versão 3.1.9.2 for Windows (Franz Faul, Universitat Kiel, Germany). Para detectar um coeficiente de correlação de 0,68 entre o tônus muscular (Escala de Ashworth) e o equilíbrio estático/ dinâmico (Escala de Berg), considerando $\alpha=5 \%$ e o poder do teste $(1-\beta)$ igual a $80 \%$, calcula-se que sejam necessários 14 indivíduos para compor a amostra do estudo. O cálculo foi realizado com base em um estudo prévio, que avaliou correlação entre equilíbrio e capacidade funcional em indivíduos hemiparéticos (NEVES et at., 2016).

Os dados foram alocados em planilha Excel e analisados no programa SPSS 25.0 (SPSS Inc., Chicago, IL, EUA). Após os procedimentos, para a análise foi utilizado o coeficiente de correlação de Spearman. A magnitude das correlações foi baseada na classificação de Munro (muito baixa = $0,00-0,25 ;$ baixa $=0,26-0,49 ;$ moderada $=$ $0,50-0,69$; alta $=0,70-0,89$; muito alta $=$ 0,90-1,00) para interpretação dos coeficientes de correlação. O nível de significância foi estabelecido em $\mathrm{p}<0,05$.

\section{Resultados}

A amostra constou com 9 homens (64,28\%) e 5 mulheres $(35,72 \%)$. Em relação à faixa etária dos participantes, compreende-se idades entre 23 e 78 anos, com uma média de idade de $59,07( \pm 14,93)$. No item que verificou o tempo médio de acometimento da doença, houve uma variação entre 6 e 228 meses, com o tempo médio de $86,57( \pm 60,67)$. Com relação ao dimídioacometido pós-AVE, o presente estudo obteve $50 \%$ de predomí- nio em cada hemicorpo. Ao verificar o tipo de AVE, obteve-se $71,43 \%$ dos indivíduos que apresentaram AVE do tipo isquêmico e $28,57 \%$ com o hemorrágico.

$\mathrm{Na}$ tabela I pode-se verificar que nela constam os escores referentes aos instrumentos de avaliação, através da escala de equilíbrio de Berg, tônus muscular pela escala de Ashworth e amplitude de movimento pela goniometria, dorsiflexionlungetest $\mathrm{e}$ flexometria do membro inferior acometido, ou seja, escores unilaterais.

Tabela I - Valores referentes aos instrumentos de avaliação, através da escala de equilíbrio de Berg, Tônus muscular pela escala de Ashworth, amplitude de movimento pela goniometria, dorsiflexionlungetest e flexometria

\begin{tabular}{lc} 
& $\begin{array}{c}\text { Média dos escores } \\
\text { (desvio padrão) }\end{array}$ \\
\hline Escala de equilíbrio de Berg & $40,71( \pm 12,06)$ \\
Tônus gastrocnêmio & $2,80( \pm 0,40)$ \\
Tônus sóleo & $2,45( \pm 0,56)$ \\
$\begin{array}{l}\text { Dorsiflexion Lunge Test cm } \\
\text { (centímetros) }\end{array}$ & $4,50( \pm 1,18)$ \\
$\begin{array}{l}\text { Dorsiflexion Lunge Test } \\
\text { '(graus) }\end{array}$ & $16,22( \pm 4,27)$ \\
Goniometria cadeia cinética & $19,07( \pm 4,33)$ \\
fechada & \\
Flexometria & $17,88( \pm 4,98)$ \\
\hline
\end{tabular}

Na tabela II estão as correlações entre a escala de equilíbrio de Berg, que apresentou o escore de 40,71( $\pm 12,06)$, com o tônus muscular, dorsiflexionlungetest (em centímetros e graus), goniometria de dorsiflexão em cadeia cinética fechada e flexometria. Observou-se que quanto maior a amplitude de tornozelo, melhor o equilíbrio o que pode ser comprovado pela significância estatística nos testes e correlação positiva com valores de $r \geq 0,472$ e $\leq 0,502$. Ressalta-se que, na amostra estudada, a não significância estatística do tônus muscular com o equilíbrio. 
Tabela II - Correlação da escala de equilíbrio de Berg com o tônus muscular e a amplitude de movimento do tornozelo, através do coeficiente de correlação de Spearman $\left(r_{s}\right)$ :

\begin{tabular}{|c|c|c|c|}
\hline & $\mathbf{r}_{\mathrm{s}}$ & Classificação & $\mathbf{p}$ \\
\hline Tônus gastrocnêmio & $-0,393$ & Baixa & 0,131 \\
\hline Tônus sóleo & $-0,384$ & Baixa & 0,136 \\
\hline $\begin{array}{l}\text { DorsiflexionLunge } \\
\text { Test (centímetros) }\end{array}$ & 0,502 & Moderada & $0,038 *$ \\
\hline $\begin{array}{l}\text { DorsiflexionLunge } \\
\text { Test (graus) }\end{array}$ & 0,488 & Baixa & $0,046^{*}$ \\
\hline $\begin{array}{l}\text { Goniometria cadeia } \\
\text { cinética fechada }\end{array}$ & 0,472 & Baixa & $0,049^{*}$ \\
\hline Flexometria & 0,490 & Baixa & $0,048^{*}$ \\
\hline
\end{tabular}

Legenda: * estatisticamente significativa, $\mathrm{p}<0,05$

\section{Discussão}

Este estudo verificou a relação entre a amplitude articular, através dos testes DorsiflexionLunge Test, goniometria e flexometria, com o equilíbrio, e foi observada correlação da amplitude de movimento do tornozelo com o equilíbrio, sendo que, quanto maior o grau de amplitude, melhor o equilíbrio. Porém, quando comparado com o tônus muscular não foi constatada tal correlação.

An e Jo (2017), com uma amostra de 26 pacientes, verificaram os efeitos da mobilização talocrural com movimento na força, mobilidade e capacidade de suporte do tornozelo em pacientes pós-AVE. Os autores referem que frequentemente esses indivíduos apresentam a dorsiflexão de tornozelo limitada, o que leva a uma transferência de peso disfuncional em direção ao membro inferior parético durante o repouso ou na marcha. $\mathrm{O}$ estudo foi dividindo em 2 grupos obtendo $12,92( \pm 7,73)$ e $8,08( \pm 6,99)$ graus como escore de dorsiflexão máxima em cadeia fechada antes da intervenção, no membro inferior não acometido. Com isso, percebe-se que quanto maior a amplitude de dorsiflexão do tornozelo, melhor o equilíbrio, pois assim o indivíduo conseguirá realizar as reações de equilíbrio, visto que a primeira estratégia está relacionada ao rolamento do tornozelo, em que o corpo desloca-se para frente, porém mantém o pé no solo.

Kime e Lee (2018) verificaram os efeitos do deslizamento posterior do tálus e a dorsiflexão do tornozelo, além de mobilização com movimento sobre o equilíbrio, pelo teste Time Upand Go e a função da marcha em pacientes com AVE crônico, em 40 participantes. Apresentaram 3,20( $\pm 2,78) \mathrm{cm}$ no DorsiflexionLunge Test e, assim, concluíram que, quanto maior a distância em centímetros, melhor o indivíduo era no teste de equilíbrio, o que se assemelha ao presente estudo, em que indivíduos que apresentaram uma maior distância durante o teste, eram os que obtiveram o melhor escore em relação a escala de Berg.

O estudo piloto de Hong et al. (2018), que avaliou quantitativamente a espasticidade pós-AVE, pela escala de Ashworth modificada, constatou uma média de 2,63 referente à espasticidade de 8 pacientes crônicos. Os autores reafirmam que a espasticidade pós -AVE é um sintoma comum, ocorrendo em aproximadamente $30 \%$ dos pacientes. O caso da espasticidade do flexor plantar do tornozelo foi observado em aproximadamente $66 \%$ dos pacientes, contribuindo, assim, consideravelmente, para deficiências que afetam o movimento do pé durante a locomoção, visto que os indivíduos que apresentaram melhor equilíbrio foram os que apresentavam um grau menor quanto à espasticidade.

No estudo de Patterson et al. (2017) foi realizada uma análise retrospectiva da pontuação da escala de Berg em indivíduos pós-AVE, numa amostra de 75 indivíduos. $\mathrm{Na}$ avaliação obteve a média de $45,7( \pm 9,3)$ de escore, mostrando-se próximo ao valor do estudo de Madhavan; Bishnoi (2017), no 
valor de 48,51 $( \pm 4,84)$ de escore, onde foi realizada a comparação do teste dos sistemas de mini-equilíbrio com a escala de equilíbrio de Berg em relação à velocidade de caminhada $\mathrm{e}$ recuperação pós-AVE em 41 pacientes, o que demonstra a correlação com o risco de queda desses pacientes. Observa-se, na amostra do presente estudo, o escore inferior aos estudos supracitados, o que sugere que estes participantes apresentam maior dificuldade para manter o equilíbrio e, consequentemente, serem mais propensos a quedas.

No estudo de Wei et al. (2017), onde foi avaliada a assimetria da marcha, espasticidade do tornozelo e depressão como preditores independentes de quedas, em 112 indivíduos pós-AVE, demonstrou por meio de avaliações (Escala de Equilíbrio de Berg, Ashwoth modificada, entre outras), que a espasticidade do gastrocnêmio era um preditor de quedas. Revelou, ainda, que o risco de queda aumentou 3,2 vezes quando a gravidade da espasticidade no gastrocnêmio aumentou em um grau. Os resultados também sustentam que a combinação de assimetria da marcha e tônus muscular anormal pode aumentar as quedas na população de AVE.
Desta maneira, Li (2017), em seu estudo sobre a espasticidade, recuperação motora e plasticidade neural após AVE, reforça que a espasticidade e a fraqueza (paresia espástica) são as principais deficiências motoras e impõem desafios significativos para o tratamento e $\mathrm{o}$ atendimento ao paciente, inclusive na obtenção do equilíbrio.O que reforça a correlação existente entre os itens avaliados. Diante da importância da amplitude de movimento de tornozelo, do tônus muscular e do equilíbrio de indivíduos pós-AVE frente à capacidade de realização de movimentos básicos e marcha, entende-se que, para essa população, deve ser incentivado o tratamento fisioterapêutico também para essa articulação.

\section{Conclusão}

Através dos resultados encontrados nesse estudo foi possível observar, na amostra estudada, que quanto maior a amplitude de dorsiflexão do tornozelo, melhor o equilíbrio. Porém, não foi constatada correlação quanto ao tônus espástico da musculatura plantiflexora e o equilíbrio.

\section{REFERÊNCIAS}

ALVES, L. X.; RANEA, R. D.; SILVA, K. R. S.; SANTOS, R. C. C. S. Correlação de déficits de equilíbrio com a independência funcional em pacientes com sequela de hemiparesia crônica pósAcidente Vascular Encefálico (AVE). UNILUS Ensino e Pesquisa, v. 35, n. 14, p. 98-99, 2017.

AN, C. M.; JO, S. O. Effects of Talocrural Mobilization with Movement on Ankle Strength, Mobility, and Weight-Bear in Ability in Hemiplegic Patients with Chronic Stroke: A Randomized Controlled Trial. Journal of Stroke and Cerebrovascular Diseases, v. 26, n. 1, p. 169-176, 2017.

BLACKBURN, M.; VAN VLIET, P.; MOCKETT, S. P. Reliability of measurements obtained with the modified Ashworth scale in the lower extremities of people with stroke. Physicaltherapy, v. 82, n. 1, p. 25-34, 2002.

BRASIL. Banco de dados do sistema único de saúde; Ministério da Saúde. Datasus. 2016. 
BROUSSY S, SAILLOUR-GLENISSON F, ROUANET F, LESAINE E, MAUGEAIS M, ALY F, et al. Sequelae and quality of life in patients living at home one year after a stroke managed in stroke units. Frontiers in Neurology, v. 10, n. 907, p. 1-7, 2019.

CHEN, C. L.; CHEN, C. Y.; CHEN, H. C.; WU, C. Y.; LIN, K. C.; HSIEH, Y. W.; SHEN, I. H. Responsiveness and minimal clinically important difference of Modified Ashworth Scale in patients with stroke. EuropeanJournalPhysicalandRehabilitation Medicine, v. 55, n. 6, p. 754-760, 2019.

DORNELLES, M. S.; OLIVEIRA, L. O.Perfil clínico e sociodemográfico de usuários com diagnóstico de acidente vascular encefálico atendidos em um laboratório de ensino prático em fisioterapia. Revista Interdisciplinar de Promoção à Saúde - RIPS, v. 2, n. 1, p. 1-7, 2019. GOUVEIA, V. H. O.; ARAÚJO, A. G. F.; MACIEL, S. S.; FERREIRA, J. J.; SANTOS, H. H. Confiabilidade das medidas inter e intra-avaliadores com goniômetro universal e flexímetro.

Fisioterapia\& Pesquisa, v. 21, n. 3, p. 229-235, 2014.

HALL, E. A.; DOUCHERTY, C. L. Validity of clinical outcome measures toe range of motion during the weigt-bearing lunge test. Journal of Science and Medicine in Sport, v. 20, n. 7, p. 618-621, 2017.

HOLT, K.; NIAZI, I. K.; NEDERGAARD, R. W.; DUEHR, J.; AMJAD, I.; SHAFIQUE, M.; ANWAR, M. N.; NDETAN, H.; TURKER, K. S.; HAAVIK, H. The effects of a single session of chiropractic care on strength, cortical drive, and spinal excitability in stroke patients. Scientific Reports, v. 9, n. 2673, p.1-10, 2019.

HONG, M. J.; PARK, J. B.; LEE, Y. J.; KIM, H. T.; LEE, W. C.; HWANG, C. M.; LIM, H. K.; LEE, D. H. Quantitative Evaluation of Post-stroke Spasticity Using Neurophysiological and Radiological Tools: A Pilot Study. Annais Rehabilitation Medicine, v. 42, n. 3, p. 384-395, 2018.

KIM, S. L.; LEE, B. H. Effects of Posterior Talar Glide and Dorsiflexion of the Ankle Plus Mobilization with Movement on Balance and Gait Function in Patient with Chronic Stroke: A Randomize Controlled Trial. Journal Neurosciences in Rural Practice, v. 9, n. 1, p. 61-67, 2018.

KIM, S. L.; LEE, B. H. The Effects of Posterior Talar Glide and Dorsiflexion of the Ankle Plus Mobilization with Movement on Balance and Gait Function in Patient with Chronic Stroke: A Randomized Controlled Trial. Journal Neurosciences in Rural Practice, v. 9, n. 1, p. 61-67, 2018.

LENDRAITIENĖ, E.; TAMOŠAUSKAITĖ, A.; PETRUŠEVIČIENĖ, D.; SAVICKAS R.

Balance evaluation techniques and physical therapy in post-stroke patients: A literature review.

NeurologiaiNeurochirurgiaPolska, v. 51, n. 1, p. 92-100, 2017.

LI S. Spasticity, Motor Recovery, and Neural Plasticity after Stroke. Frontiers in Neurology, v. 8, n. 20, p. 1-8, 2017.

MADHAVAN S, BISHNOI A. Comparison of the Mini-Balance Evaluations Systems Test with the Berg Balance Scale in relationship to walking speed and motor recovery post stroke. Topics Stroke Rehabilitation, v. 24, n. 8, p. 579-584, 2017.

MATSUDA F, MUKAINO M, OHTSUKA K, TANIKAWA H, TSUCHIYAMA K, TERANISHI T, et al. Biomechanical factors behind toe clearance during the swing phase in hemiparetic patients. Topics in strokerehabilitation, v. 24, n. 3, p. 177-182, 2017.

MEDEIROS, C. S. P.; FERNANDES, S. G. G.; SOUZA, D. E.; GUEDES, D. T.; CACHO, E. W. A.; CACHO, R. O. Comprometimento motor e risco de quedas empacientes pós-acidente vascular encefálico. RevistaBrasileira de Ciências. eMovimento, v. 27, n. 1, p. 42-49, 2019.

MEULEN, F. B. V.; WEENK, D.; ASSELDONK, E. H. F.; SCHEPERS, H. M.; VELTINK, P. H.; BUURKE, A. J. H. Analysis of balance during functional walking in stroke survivors. PlosOne, v. 11, n. 11, p. 162-169, 2016.

MINISTÉRIO DA SAÚDE. Diretrizes de Atenção à Reabilitação da Pessoa com Acidente 
Vascular Cerebral. Brasília: Secretaria de Atenção à Saúde. Departamento de Ações Programáticas Estratégicas, Ministério da Saúde. 1. ed. 72p, 2013.

MIYAMOTO, S. T.; LOMBARDI, J. I.; BERG, K. O.; RAMOS, L. R.; NATOUR, J. Brazilian version of the Berg balance scale. BrazilianJournalof Medical BiologicalResearch, v. 37, n. 9, p. 1411-1421, 2004.

MOLITERNO, A. H.; PADOVAN, B. M.; VIANA, J. S.; PEREIRA, A. S.; GUERRERO, K. M.; GONZAGA, C. N.; DAMASCENO, S. O.; FRASSON, I. B.; TACAO, G. Y.; BARBATTO, L. M.; LORENÇONI, R. M. R.; CARVALHO, C. O desempenho da marcha de hemiparéticos crônicos e a correlação com a recuperação e o nível de comprometimento motor funcional. Colloquium Vitae, v. 11, n. 1, p. 63-69, 2019.

NEVES J.; FERREIRA G.; CONCEIÇÃO T. Relationship between the balance and functional capacity in hemiparetics. RevistaBrasileira de Neurologia, v. 52, n. 2, p. 1231-1236, 2016.

PATTERSON, K. K.; INNESS, E.; MCILROY, W. E.; MANSFIELD, A. A Retrospective Analysis of Post-Stroke Berg Balance Scale Scores: How Should Normal and At-Risk Scores Be Interpreted? Physiotherapy Canada, v. 69, n. 2, p. 142-149, 2017.

SAVASSINI, D. J.; HELL, F. R.; SPINIELI, R. L.; LIRA, J. J. Sistema nervoso se regenera? A neuroplasticidade na reabilitação de hemiparesia decorrente de ave. Edição especial: vi ciclo científico. RevistaSaberes da Faculdade São Paulo, v. 10, n. 1, p. 1-12, 2019.

SONG, M. J.; LEE, J. H.; SHIN, W. S. Minimal clinically important difference of Berg balance scale scores in people with agude stroke. Physical Therapy Rehabilitation Science, v. 7, n. 3, p. 102$108,2018$.

SZOPA, A.; DOMAGALSKA-SZOPA, M.; LASEK-BAL, A.; ŻAK, A. The link between weight shift asymmetry and gait disturbances in chronic hemiparetic stroke patients. Clinical Interventions in Aging, v.12, p. 2055-2062, 2017.

WEI, T. S.; LIU, P. T.; CHANG, L. W.; LIU, S. Y. Gait asymmetry, ankle spasticity, and depression as independent predictors of falls in ambulatory stroke. PlosOne, v. 12, p. 5, p. 1-14, 2017. 
